\title{
Depresión en pacientes adultos mayores de comunidades de altura, con enfermedad pulmonar obstructiva crónica (EPOC).
}

Depression in elderly patients from highland communities with chronic obstructive pulmonary disease (COPD).

Valeria A. Castro-Benites ${ }^{1}$, Fernando M. Runzer-Colmenares ${ }^{1,2}$, José F. Parodi ${ }^{2}$

\section{RESUMEN}

Objetivo: Determinar la frecuencia de depresión y características sociodemográficas en pacientes adultos mayores con enfermedad pulmonar obstructiva crónica (EPOC), viviendo en zonas de altura. Métodos: Estudio retrospectivo de carácter descriptivo en 16 pacientes con EPOC, de 60 o más años de edad, habitantes del altiplano andino $(\geq 1500$ $\mathrm{m}$ sobre el nivel del mar). Se analizaron las características sociodemográficas, nivel cognitivo, funcional, emocional y antecedentes personales de cada uno de los probandos. Resultados: El promedio de edad de los pacientes fue 72,1 años (DE 5,37), el 62,5\% fueron mujeres, el 75\% provenían de áreas rurales y el $81,2 \%$ eran analfabetos o tenían solo instrucción primaria incompleta. Entre las características clínicas, el 18,7\% presentaban hipertensión arterial, el $12,5 \%$ presentaron diabetes mellitus tipo 2 y el $87,5 \%$ reportó no consumir tabaco. De acuerdo al Test de Yesavage, el 43,7\% presentaban depresión y el 56,2\% refirieron sentirse frecuentemente deprimidos. Conclusiones: Este estudio sugiere una alta frecuencia de depresión en pacientes con EPOC que habitan regiones de la altura andina.

PALABRAS CLAVE: EPOC, adulto mayor, Andes, depresión.

\section{SUMMARY}

Objective: To determine the frequency of depression and sociodemographic characteristics of elderly patients with chronic obstructive pulmonary disease (COPD), living in zones of high altitude. Methods: A retrospective and descriptive study carried out in 16 patients of 60 years of age or older, inhabitants of the highlands $(\geq 1500$ meters above sea level) in the Peruvian Andes, with the diagnosis of COPD. Sociodemographic, cognitive, functional and emotional characteristics of each of the probands were assessed. Results: The average age of the sample of patients was 72.1 (SD 5.37), $62.5 \%$ of them were women, $75 \%$ came from rural areas, and $81.2 \%$ were illiterate or had only elementary education. Among the clinical features, $18.7 \%$ had arterial hypertension, $12.5 \%$ had type 2 diabetes mellitus, and $87.5 \%$ reported no tobacco consumption. According to the Yesavage test, $43.7 \%$ had depression; and $56.2 \%$ reported being frequently depressed. Conclusion: This study provides evidence of a high prevalence of depression in COPD patients living in high-altitude zones.

KEY WORDS: COPD, elderly, Andes, depression.

1 Universidad Científica del Sur. Lima, Perú.

2 Universidad de San Martín de Porres, Facultad de Medicina Humana, Centro de Investigación del Envejecimiento (CIEN). Lima, Perú. 
Depresión en pacientes adultos mayores de comunidades de altura, con enfermedad pulmonar obstructiva crónica.

\section{INTRODUCCIÓN}

La enfermedad pulmonar obstructiva crónica (EPOC) es una entidad prevalente en nuestro medio, se estima que 64 millones de personas sufren de esta condición en el mundo y para el 2030 será la cuarta causa de muerte (1).

La morbi-mortalidad ha ido incrementando sostenidamente a un ritmo más lento que en décadas anteriores e inclusive ha disminuido en hombres. Casi el $90 \%$ de las muertes relacionadas a EPOC ocurren en los países subdesarrollados o en vías de desarrollo con una gran carencia económica y social (2).

En Latinoamérica, el estudio PLATINO (Proyecto Latinoamericano de Investigación en Obstrucción Pulmonar) evaluó la prevalencia de EPOC en países latinoamericanos que fue de 19,7\% para Montevideo, $16,9 \%$ para Santiago, 15,8\% para San Pablo, $12,1 \%$ para Caracas y $7,8 \%$ para México (3).

Por otro lado, en el mundo, alrededor de 400 millones de personas viven en zonas de altura (por encima de los 1500 m s.n.m.), habiendo una prevalencia mayor de pacientes por EPOC en dichos lugares (4). Los escasos recursos y el difícil acceso a la salud dificultan el diagnóstico y manejo de dicha enfermedad, así como los factores desencadenantes y exacerbantes. En los últimos años se ha investigado diferentes causas para las exacerbaciones, hospitalizaciones, índice de comorbilidades, su impacto y muerte, encontrando depresión como factor de riesgo.

Diferentes estudios han demostrado que los trastornos mentales son tres veces más prevalentes en personas con EPOC (5). En un reciente metaanálisis se encontró que 1 de cada 4 pacientes con EPOC presentan signos clínicos de depresión (6). Así mismo, otros estudios sugieren que la prevalencia de depresión varía entre 10 a $42 \%$ de los pacientes y esto podría ser un agravante de la enfermedad y tendría relación con la tasa de mortalidad. Por otro lado, se necesitan más estudios para evaluar el impacto que tiene la etnia y la nacionalidad con la prevalencia de depresión en EPOC ya que en los pocos estudios que existen se ha visto su discordancia (7).

Es por ello que es importante conocer e identificar a los pacientes con depresión y EPOC, realizando un diagnóstico correcto. El objetivo del presente estudio fue determinar la frecuencia de depresión en adultos mayores con EPOC residentes en comunidades altoandinas, tomando en cuenta sus características sociodemográficas.

\section{MATERIAL Y MÉTODOS}

Este es un estudio descriptivo, retrospectivo, producto de un análisis secundario de bases de datos realizado en habitantes de 60 años o más, que viven en el altiplano andino $(\geq 1500 \mathrm{~m}$ sobre el nivel del mar). Las comunidades del Perú que se tomaron para el estudio fueron: La Jalca, Leimebamba (Departamento de Amazonas), Llupa, San Pedro de Chaná, Atipayan (Departamento de Áncash), Pampamarca (Departamento de Huánuco), Chacapampa (Huancayo), Ayahuanco (Departamento de Ayacucho), Paucarcolla (Departamento de Puno), Vilca (Departamento de Huancavelica) y Viñac (Lima) entre 2013 y 2016. Los habitantes de las 11 comunidades tenían en común el mismo grupo étnico y trabajo el cual era agricultura, ganadería y comercio.

Descripción del área de estudio: Las comunidades fueron localizadas en los Andes peruanos: a) La Jalca: asentamiento urbano localizado a 2800 m s.n.m.; b) Leimebamba: pueblo rural localizado a $2158 \mathrm{~m}$ s.n.m.; c) Llupa: pueblo rural localizado a $3511 \mathrm{~m}$ s.n.m.; d) San Pedro de Chaná: pueblo rural localizado a 3413 m s.n.m.; e) Atipayán: pueblo rural localizado a 3364 m s.n.m.; f) Pampamarca: pueblo rural localizado a 3445 m s.n.m.; g) Chacapampa: pueblo rural localizado a 3358 m s.n.m.; h) Ayahuanco: pueblo rural localizado a 3414 m s.n.m.; i) Paucarcolla: pueblo rural localizado a 3847 m s.n.m.; j) Vilca: pueblo rural localizado a 3275 m s.n.m.; k) Viñac: pueblo rural localizado a 3315 m s.n.m..

Es un estudio con muestreo no probabilístico en el que se realizó un muestreo tipo censo registrando a todos los adultos mayores de 60 años pertenecientes a las comunidades altoandinas ya mencionadas. La muestra fue de 413 adultos de los cuales solo se trabajó con aquellos que presentaban EPOC siendo la muestra final de 16 pacientes.

Para la realización del este estudio se tomaron como criterios de selección los siguientes: adultos de 60 años a más con diagnóstico de EPOC en las comunidades del Altiplano que vivan por encima de 1500 m s.n.m., que firmen el consentimiento informado, con función neurológica conservada y sin deterioro cognitivo grave. Se consideró como EPOC a la respuesta positiva del paciente o familiar a la pregunta: “¿algún médico o personal de salud le ha informado que 
sufre de enfermedad pulmonar obstructiva crónica?" o a la presencia de dicho diagnóstico en la cartilla de Valoración Clínica del Adulto Mayor (VACAM) propuesto por el Ministerio de Salud el Perú (MINSA).

Los participantes fueron visitados en sus casas hasta 3 veces para invitarlos a participar del estudio. Aquellos que aceptaron, debieron firmar un consentimiento informado previo a la recolección de información. La información recolectada fue clasificada según características sociodemográficas, antecedente médico, estado funcional y estado cognitivo y psicológico. La entrevista fue realizada por un médico geriatra, médicos y estudiantes de medicina (entrenados previamente por el médico geriatra).

Se utilizó STATA v14.0 para el análisis estadístico de este estudio. Los resultados descriptivos fueron presentados usando medidas de tendencia central, frecuencias absolutas y relativas y medidas de dispersión. Las características sociodemográficas fueron incluidas y evaluadas por autoevaluación: edad (categorizado en menor de 70 años de edad, entre 71 y 80 años y mayor de 80 años), género (femenino/masculino), educación (analfabeto, primaria incompleta, primaria completa, estudios secundarios completos), estado civil (soltero/soltera, casado/casada, viudo-a/divorciado-a), tipo de poblado (rural/urbano). La información sociodemográfica fue corroborada con el documento nacional de identidad (DNI) de cada participante.

Las siguientes variables fueron evaluadas por auto reporte: consumo de tabaco (sí o no), hipertensión arterial (HTA) (si o no), diabetes mellitus II (DM2) (sí o no), índice de masa corporal (IMC, en $\mathrm{kg} / \mathrm{m} 2$ ). Se ha categorizado a la población en: malnutrición $(<18,5)$, normal (entre 18,5-24,99), sobrepeso (entre $25,0-29,99)$ y obesidad $(>30)$.

También se evaluó la percepción de los pacientes sobre su propia salud con las siguientes preguntas: "Diría que su salud es: ¿Excelente?, ¿Muy buena?, ¿Buena?, ¿Regular?, ¿Mala?”

Para la valoración funcional incluimos la velocidad de marcha independientemente de la batería de rendimiento físico corto.

Se usó el Índice de Barthel, un cuestionario sobre 10 actividades básicas de la vida diaria (ABVD) con puntuación total de 0 a 100 . Las personas que no obtuvieron un puntaje de 100 fueron clasificadas como dependientes para actividades básicas de la vida diaria.

Se utilizó el cuestionario de Pfeiffer, un cuestionario de 10 preguntas para evaluar deterioro cognitivo. Los estratos se generaron de la siguiente manera: sin deterioro (0-2 errores), deterioro leve (3-4 errores), moderado deterioro (5-7 errores) y deterioro grave (8-10). Aquellos con 8 o más errores fueron excluidos (8).

La depresión se definió según el cuestionario de Yesavage versión de 5 ítems en donde se cuestionó: 1) ¿está usted satisfecho con la vida que lleva?, 2) ¿siente que su vida está vacía?, 3) ¿siente que está aburrido frecuentemente?, 4) ¿está preocupado porque piensa que algo malo le va a pasar?, 5) ¿piensa que la mayoría de gente tiene más suerte que usted? Se considera depresión al obtener 3 puntos o más. Adicionalmente, se utilizó una pregunta independiente del Test de Edmonton de Fragilidad la cual fue respecto al ánimo: "Se siente frecuentemente triste o deprimido?" Respondiendo sí o no.

El protocolo fue evaluado y aprobado por el Comité de Ética de la Universidad Científica del Sur.

\section{RESULTADOS}

Se analizaron los datos de los 16 participantes con EPOC que respondieron completamente a la encuesta. Se encontró que el promedio de edad de estos pacientes fue de 72,1 años con una desviación estándar (DE) de 5,37 ; el $62,5 \%$ de ellos fueron mujeres.

Con respecto al estado civil, el 50\% eran casados dividiéndose el otro $50 \%$ entre solteros y divorciados; no se encontró ningún viudo. El $75 \%$ de los participantes provenían de área rural, solo 4 vivían en la zona urbana y la mayoría, el $81,2 \%$ eran analfabetos o tenían primaria incompleta (tabla 1).

Con respecto a las características clínicas, se quiso evaluar si estos pacientes presentaban otra comorbilidad a parte del EPOC evidenciando que solo el $18,7 \%$ de los encuestados presentan hipertensión arterial y el $12,5 \%$ presentan diabetes mellitus tipo 2. Por otro lado, el $87,5 \%$ de los encuestados dijo no consumir tabaco lo que lleva a pensar en otras posibles causas de EPOC. El promedio de índice de masa corporal fue de 26,02 con una DE de 3,3 evidenciando un ligero sobrepeso en los pacientes encuestados. 
Depresión en pacientes adultos mayores de comunidades de altura, con enfermedad pulmonar obstructiva crónica.

Tabla 1. Características sociodemográficas de los participantes del estudio $(\mathrm{n}=16)$.

\begin{tabular}{lccc}
\hline Variables & $\mathbf{n}$ & $\mathbf{\%}$ & media $\pm \mathbf{D E}$ \\
\hline Datos socio-demográficos & & & \\
Sexo & 10 & 62,5 & \\
$\quad$ Femenino & 6 & 37,5 & \\
$\quad$ Masculino & & & $72,13 \pm 5,37$ \\
Edad en años & & & \\
Tipo de poblado & 4 & 25 & \\
$\quad$ Urbano & 12 & 75 & \\
$\quad$ Rural & & & \\
Estado civil & 4 & 25 \\
$\quad$ Soltero (a) & 8 & 50 \\
$\quad$ Casado (a) & 0 & 0 & \\
$\quad$ Viudo (a) & 4 & 25 \\
$\quad$ Divorciado / separado (a) & & \\
Grado de instrucción & 13 & 81,2 \\
$\quad$ Analfabeto o primaria incompleta & 3 & 18,7 \\
$\quad$ Primaria completa &
\end{tabular}

Tabla 2. Características clínicas de los participantes del estudio $(\mathrm{n}=16)$.

\begin{tabular}{lccc}
\hline Variables & $\mathbf{n}$ & $\mathbf{\%}$ & media $\pm \mathbf{D E}$ \\
\hline Datos & & & \\
Hipertensión arterial & & & \\
$\quad$ No & 13 & 81,2 & \\
$\quad$ Sí & 3 & 18,7 & \\
Diabetes Mellitus tipo 2 & & & \\
$\quad$ No & 14 & 87,5 & \\
$\quad$ Sí & 2 & 12,5 & \\
Consumo de Tabaco & & & \\
$\quad$ No & 14 & 87,5 & \\
$\quad$ Sí & 2 & 12,5 & \\
Índice de Masa Corporal & & & $26,02 \pm 3,3$ \\
Índice de Barthel & & & $86,5 \pm 12,2$ \\
Cuestionario de Pfeiffer & & & $1,81 \pm 1,9$ \\
Velocidad de la marcha & & & $0,7 \pm 0,3$ \\
\hline
\end{tabular}

Al evaluar el área funcional y cognitiva, se evidenció que la mayoría de los pacientes presentaban un ligero grado de dependencia teniendo como promedio el Índice de Barthel de 86,5 con DE de 12,2 y una función intelectual intacta teniendo como resultado un Test de Pfeiffer promedio de 1,81 con DE de 1,9. La velocidad de la marcha promedio fue de 0,7 $\mathrm{m} / \mathrm{s}$ con DE de 0,3 (tabla 2). El 25\% de la población encuestada dijo vivir solo; en cuanto a su percepción de la salud, el 37,5\% respondió que siente tener una salud regular seguido del $31,2 \%$ que refirió tener mala salud. Para evaluar depresión, los encuestados 
Tabla 3. Características sociales y salud mental de los participantes del estudio $(\mathrm{n}=16)$.

\begin{tabular}{lcc}
\hline Variables & $\mathbf{n}$ & $\mathbf{\%}$ \\
\hline Datos & & \\
Vive solo & & \\
$\quad$ No & 12 & 75 \\
$\quad$ Sí & 4 & 25 \\
Autopercepción de la salud & & \\
$\quad$ Excelente & 2 & 12,6 \\
$\quad$ Buena & 3 & 18,7 \\
$\quad$ Regular & 6 & 37,5 \\
$\quad$ Mala & 5 & 31,2 \\
Depresión (según Cuestionario de Yesavage) & & \\
$\quad$ No & 9 & 56,2 \\
$\quad$ Sí & 7 & 43,7 \\
Auto reporte de depresión & & \\
$\quad$ No & 7 & 43,7 \\
$\quad$ Sí & 9 & 56,2 \\
\hline
\end{tabular}

respondieron el cuestionario de Yesavage encontrando que el $43,7 \%$ sí presentan depresión y el 56,2\% refirió sentirse frecuentemente deprimido (tabla 3 ).

\section{DISCUSIÓN}

Se ha visto que la prevalencia de depresión en pacientes estables con EPOC va de 10\% a 57\% (9), porcentaje que concuerda con este estudio ya que entre los 16 pacientes con EPOC de las 11 comunidades altoandinas, el 43,7\% presentaban depresión según el Cuestionario de Yesavage y el 56,2\% refirió sentirse triste la mayor parte del tiempo.

En cuanto al género, en nuestro estudio observamos que el $62,5 \%$ eran mujeres con EPOC y depresión, resultados que se relacionan con diversos estudios los cuales concuerdan que las mujeres con EPOC tienen mayor probabilidad de presentar depresión respecto a los hombres así como mayor severidad $(10,11)$.

Entre los factores de riesgo para EPOC, el más importante es el consumo de tabaco que está íntimamente relacionado con depresión ya que ellos tienen más probabilidad de fumar, tienen mayor riesgo de hacer dependencia y se les dificulta más cesarlo $(12,13)$. A pesar de que varios estudios concuerdan que los fumadores son más propensos a deprimirse (14), en este estudio se vio que solo el $12,5 \%$ de los encuestados consumía tabaco. Esto nos lleva a pensar que lo más probable es que la causa de EPOC en estos pacientes no sea el tabaco sino su prolongada exposición a agentes de biomasa.

Con respecto al vivir en zonas de gran altura, un estudio realizado en ratas hembras evidenció que dejarlas en la altura durante una semana puede aumentar sus manifestaciones depresivas sugiriendo que la altitud sola puede empeorar el estado de depresión, probablemente debido a la hipoxia hipobárica crónica (15). La hipoxia en ratas provocada por la altura puede causar alteración de los metabolitos del lóbulo frontal exacerbando el comportamiento depresivo (16). Además, se ha evidenciado que la exposición durante 1 a 2 semanas a la hipoxia hipobárica provoca niveles bajos de serotonina siendo esta otra posible causa del aumento del comportamiento depresivo (17). Existe muy escasa evidencia acerca de la fisiopatología de trastornos del ánimo en pacientes adultos mayores con EPOC residentes en zonas de altura.

En general, los pacientes con depresión perciben que su salud está más deteriorada con respecto a la población promedio (18). Un metaanálisis mostró que la presencia de depresión entre los pacientes con EPOC fue una de las correlaciones más fuertes con la autopercepción de su estado de salud (19). 
Depresión en pacientes adultos mayores de comunidades de altura, con enfermedad pulmonar obstructiva crónica.

En este estudió se analizó también la percepción que tenían los encuestados sobre su salud, donde el $37,5 \%$ refirió sentir que tiene una salud regular seguido por el $31,2 \%$ que refirió tener una mala salud, datos que concuerdan con los estudios ya nombrados.

Martínez et al (20) estudiaron el desarrollo de la discapacidad en pacientes con EPOC y determinaron que el $12,8 \%$ de los pacientes con EPOC presentaban algún tipo de discapacidad volviéndose dependientes y que esto tenía una estrecha relación con el deterioro cognitivo progresivo. En nuestro estudio se valoró el área funcional así como la cognitiva: los encuestados tenían un promedio de 86,5 en el Índice de Barthel concluyendo que la mayoría tenía un grado de dependencia leve y un nivel cognitivo intacto con un resultado del Cuestionario de Pfeiffer de 1,81. Si se trata la enfermedad pulmonar y el área emocional podemos evitar una progresión más rápida de la enfermedad, reducir comorbilidades y disminuir la tasa de mortalidad. Por otro lado, este estudio también encontró que el $81,2 \%$ de los encuestados eran analfabetos o no habían terminado la escuela primaria. La misma evidencia se encontró en el estudio de Betolaza et al., en donde hallaron que la mayoría de encuestados tenían un bajo nivel de instrucción correspondiendo al 35,7\% (21).

$\mathrm{Al}$ analizar las comorbilidades de los encuestados se vio que de los 16, el 18,7\% presentaba hipertensión arterial; y el $12,5 \%$, diabetes mellitus tipo 2. Es probable que en nuestro estudio esta relación no sea muy significativa ya que la muestra es reducida a diferencia de Brodnicka et al., quienes encontraron comorbilidades en el 95\% de los pacientes (22), y de Kupcewicz y Abramowicz que las hallaron en el 40\% (23). Además, en el estudio de Antonelli Incalzi et al., realizado en un grupo de 270 pacientes con EPOC el $28 \%$ presentaba hipertensión, el $14 \%$ diabetes y el $10 \%$ enfermedad coronaria (24).

En el estudio de Zaeh et al., analizaron la relación que existe en pacientes con enfermedades pulmonares crónicas que viven en la altura de Perú con depresión (25). Ellos concuerdan, junto con nosotros, que sí hay estrecha relación entre depresión y la altitud y que la mayoría de pacientes son mujeres. Pero, a diferencia de nuestro estudio, su estudio fue realizado en pacientes mayores de 35 años, teniendo una edad de alrededor de los 50 años. Por lo tanto, hasta donde conocemos, este estudio es el primero en investigar la frecuencia de depresión en pacientes adultos mayores del altiplano con EPOC.
El presente estudio cuenta con algunas limitaciones. El tamaño de muestra es pequeño y, dado que los antecedentes médicos fueron incluidos como autoreporte o por reporte de familiares, se pudo haber incurrido en sesgo de información, más aún en una población con un porcentaje alto de analfabetismo. Sin embargo, se trató de utilizar herramientas de evaluación basadas en la VACAM propuesta por el MINSA, validadas para su uso en atención primaria.

En conclusión, este estudio proporciona evidencia de una alta frecuencia de depresión en pacientes con EPOC, posiblemente la vida a gran altura sea un factor de riesgo potencialmente importante, o en su defecto, los condicionantes sociales podrían tener impacto. A pesar de contar con una muestra pequeña, creemos que este estudio puede motivar a otros profesionales a darle la debida importancia e investigar más sobre este tema que actualmente es muy poco difundido, con el fin de mejorar la calidad de vida de estos pacientes, informar al personal de salud y lograr mejores resultados de salud en general.

\section{Correspondencia}

Valeria Castro

Correo electrónico: vale_ji27@hotmail.com

Financiamiento y conflictos de interés: Este trabajo de investigación fue autofinanciado. Los autores no declaran tener conflictos de intereses.

\section{REFERENCIAS BIBLIOGRÁFICAS}

1. Vestbo J, Hurd SS, Agustí AG, Jones PW, Vogelmeier C, Anzueto A, et al. Global strategy for the diagnosis, management, and prevention of chronic obstructive pulmonary disease: GOLD executive summary. Am J Respir Crit Care Med. 2013; 187(4):347-65.

2. Public Health Agency of Canada. Chronic Pulmonary Obstructive Disease (COPD). Ottawa: Public Health Agency of Canada; 2011.(Fecha de acceso 24 de marzo del 2016) Disponible en: http://www.phacaspc.Gc.ca/cd-mc/publications/copd-mpoc/ ff-rr2011-eng.php

3. Menezes AMB, Perez-Padilla R, Jardim JRB, Muiño A, Lopez MV, Valdivia G, et al. Chronic obstructive pulmonary disease in five Latin American cities (the PLATINO study): a prevalence study. Lancet. 2005; 366:1875-81.

4. Brakema E, Tabyshova A, Kasteleyn M. High COPD prevalence at high altitude: does household air pollution play a role? Eur Respir J. 2018; 10(1183): 1193-2018.

5. Bock K, Bendstrup E, Hilberg O, Løkke A. Screening 
tools for evaluation of depression in Chronic Obstructive Pulmonary Disease (COPD). A systematic review. Eur Clin Respir J. 2017; 4(1):1332931.

6. Zhang MW, Ho RC, Cheung MW, Fu E, Mak A. Prevalence of depressive symptoms in patients with chronic obstructive pulmonary disease: a systematic review, meta-analysis and meta-regression. Gen Hosp Psychiatry. 2011;33(3):217-223.

7. Panagioti, M, Scott C, Blakemore A, Coventry PA. Overview of the prevalence, impact, and management of depression and anxiety in chronic obstructive pulmonary disease. Int J Chron Obstruct Pulmon Dis. 2014; 9: 1289-1306.

8. Urrunaga-Pastor D, Moncada-Mapelli E, RunzerColmenares FM, et al. Factors associated with poor balance ability in older adults of nine high-altitude communities. Arch Gerontol Geriatr. 2018; 77:108114.

9. Pumar MI, Gray CR, Walsh JR, Yang IA, Rolls TA, Ward DL. Anxiety and depression-Important psychological comorbidities of COPD. J Thorac Dis. 2014; 6(11):1615-31.

10. Dowson C, Laing R, Barraclough R, et al. The use of the Hospital Anxiety and Depression Scale (HADS) in patients with chronic obstructive pulmonary disease: a pilot study. N Z Med J. 2001; 114: 447-9.

11. Hanania NA, Mullerova H, Locantore NW, et al. Determinants of depression in the ECLIPSE chronic obstructive pulmonary disease cohort. Am J Respir Crit Care Med. 2011; 183:604-11.

12. Glassman AH, Helzer JE, Covey LS, et al. Smoking, smoking cessation, and major depression. JAMA. 1990; 264:1546-9.

13. Glassman AH, Covey LS, Stetner F, et al. Smoking cessation and the course of major depression: a follow-up study. Lancet. 2001; 357:1929-32.

14. Wiesbeck GA, Kuhl HC, Yaldizli O, et al. Tobacco smoking and depression--results from the $\mathrm{WHO} /$ ISBRA study. Neuropsychobiology. 2008; 57:26-31.

15. Kanekar S, Bogdanova OV, Olson PR, Sung YH, D'Anci KE, Renshaw PF. Hypobaric hypoxia induces depression-like behavior in female Sprague-Dawley rats, but not males. High Alt Med Biol. 2015; 16: $52-60$.
16. Bogdanova O, Abdullah O, Kanekar S, Bogdanov V, Prescot A, Renshaw P. Neurochemical alterations in frontal cortex of the rat after a week of hypobaric hypoxia. Behav Brain Res. 2014; 263: 203-209.

17. Ray K, Dutta A, Panjwani U, Thakur L, Anand J P, Kumar S. Hypobaric hypoxia modulates brain biogenic amines and disturbs sleep architecture. Neurochem Int. 2011; 58: 112-118.

18. Wells KB, Golding JM, Burnam MA. Psychiatric disorder and limitations in physical functioning in a sample of the Los Angeles general population. Am J Psychiatry. 1988; 145:712-7.

19. Tsiligianni I, Kocks J, Tzanakis N, et al. Factors that influence disease-specific quality of life or health status in patients with COPD: a review and metaanalysis of Pearson correlations. Prim Care Respir J. 2011; 20:257-68.

20. Martinez CH, Richardson CR, Han MK, Cigolle CT. Chronic obstructive pulmonary disease, cognitive impairment, and development of disability: the health and retirement study. Ann Am Thorac Soc. 2014; 11(9):1362-1370.

21. Betolaza S, Correa S, Gonzales M. Estudio descriptivo de una población de pacientes EPOC asistidos en el Hospital Pasteur: severidad e impacto en la vida diaria. Rev Urug Med Interna. 2018; 2(13): 13-21.

22. Brodnicka I, Nowak-Gabryel M, Gabryel M, Grabicki M. Dependence of comorbidities in chronic obstructive pulmonary diseases (COPD) on the age of patients and on the severity of COPD. Med News. 2010;79(5):362-368.

23. Kupcewicz E, Abramowicz A. Assessment of quality of life in chronic obstructive pulmonary disease patients. Hygeia Public Health. 2014;49(4):805-812.

24. Antonelli R, Fuso L, De Rosa M, et al. Co-morbidity contributes to predict mortality of patients with chronic obstructive pulmonary disease. Eur Respir J. 1997; 10(12): 2794-2800.

25. Zaeh S, Miele H, Putcha N, et al. Chronic respiratory disease and high altitude are associated with depressive symptoms in four diverse settings. Int $\mathrm{J}$ Tuberc Lung Dis. 2016;20(9): 1263-1269.

Recibido: 31/01/2019

Aceptado: 10/03/2019 